\title{
Efeitos da introdução do PAB sobre a distribuição de recursos e a prestação de serviços: o caso de Minas Gerais
}

\author{
Assessing the effect of the PAB equity initiative \\ on resource allocation and service inequalities \\ among municipalities in Minas Gerais - Brazil
}

Edite Novais da Mata Machado 1

Fátima Beatriz Carneiro Teixeira P. Fortes 1

Mercês Somarriba 2

\footnotetext{
1 Centro de Estudos

Econômicos e Sociais, Fundação João Pinheiro. Alameda das Acácias 70, 31275-150, Belo Horizonte MG.edite@fjp.gov.br

2 Pesquisadora visitante na Fundação João Pinheiro com apoio da FAPEMIG.
}

\begin{abstract}
The project aimed at verifying whether the Basic Health Care Floor (PAB) would have favored a reduction of inequalities (funding and use of services) among the municipalities of an economic and social heterogeneous state of the Brazilian federation. The increase of resources applied to basic health care, occurred with the PAB introduction, has made possible a redistribution of resources favorable to the poorest region in the state and to the smaller population municipalities. Actually, the variation of the per capita values of the federal transfers to basic health care between 1997 and 2000 presented itself negatively associated to the production of health services and to the municipalities' expenditure capacity in 1997. The correlation between the municipal values of the per capita PAB in 1997 and 2000 and the variables that reflect health need showed that the changes introduced leaned towards benefiting the municipalities with greater health need, which could considered pro-equity.
\end{abstract}

Key words Inequalities, Equity, Resource allocation, Basic health care, $P A B$
Resumo O foco deste estudo foi a avaliação dos efeitos da introdução do Piso de Atenção Básica (PAB) na distribuição de recursos e na prestação de serviços no âmbito da atenção básica entre municípios de Minas Gerais. A introdução do PAB foi acompanhada de elevação dos recursos federais transferidos para atenção básica, mas a distribuição dos recursos federais entre municípios se manteve desigual. As mudanças favoreceram os municípios de pequeno porte populacional e o Vale do Jequitinhonha, a região mais pobre do Estado. A variação dos valores per capita das transferências federais para atenção básica entre 1997 e 2000 mostrou-se negativamente associada à produção de serviços de saúde e à capacidade de gasto dos municípios em 1997. A correlação entre os valores municipais do PAB per capita de 1997 e 2000 e as variáveis que refletem necessidades em saúde mostrou, por sua vez, que as mudanças tenderam a beneficiar os municípios com maior necessidade em saúde, podendo ser consideradas pró-eqüidade.

Palavras-chave Desigualdade, Eqüidade, Distribuição de recursos, Atenção básica à saúde, $P A B$ 


\section{Introdução}

Este artigo apresenta os resultados da avaliação dos efeitos da introdução do Piso de Atenção Básica (PAB) sobre a distribuição de recursos e a prestação de serviços no âmbito da atenção básica, entre municípios do Estado de Minas Gerais (Fundação João Pinheiro, 2003). A pesquisa financiada pela Aliança para Pesquisa em Políticas e Sistemas de Saúde, uma iniciativa do Fórum Global para Pesquisa em Saúde, em colaboração com a Organização Mundial da Saúde, foi concebida como projeto destinado a fortalecer a capacidade de formulação de políticas, na linha de pesquisa denominada research for policy. Buscou analisar dados quantitativos disponíveis sobre distribuição de recursos e uso de serviços, bem como discutir problemas vividos concretamente pelos responsáveis pela política de saúde, nas esferas estadual e municipal. Pretendeu-se, com isso, realizar uma avaliação que, além de tentar quantificar resultados e impactos da distribuição de recursos sobre o acesso a serviços, buscasse compreender fatores ligados à capacidade institucional das esferas estadual e municipal mais ou menos favoráveis à efetiva implementação dos princípios do SUS, principalmente no que se refere à busca de eqüidade e de integralidade da atenção à saúde. Neste sentido, o presente estudo se filia à proposta de avaliação processual da política de saúde que ressalta a importância de se identificar o contexto de implantação dos programas e intervenções pelas variáveis-chave como capacidade técnica e administrativa das instâncias estadual e municipal, relações intergovernamentais, compromisso com metas por parte dos gestores municipais, grau de dificuldade dos municípios menores e mais pobres para a contratação de profissionais qualificados, entre outras (Bodstein, 2002).

No desenvolvimento da pesquisa, distintas estratégias metodológicas foram adotadas. Inicialmente foi realizada uma análise quantitativa que buscou identificar as mudanças na distribuição dos recursos federais e dos serviços de atenção básica antes e após o PAB. Complementarmente, abordagem qualitativa envolveu entrevista a atores relevantes localizados em áreas estratégicas da Secretaria de Estado da Saúde de Minas Gerais (SES-MG), nas Secretarias de Saúde de cinco municípios selecionados para estudos de caso, e no colegiado de Secretários Municipais de Saúde (COSEMS-MG). A Comissão Intergestores Bipartite (CIB) foi abordada por meio de consulta às atas de suas reuniões. Na pesquisa de campo nos cinco municípios foram levantados dados quantitativos e qualitativos sobre condições socioeconômicas e serviços de saúde.

O trabalho está organizado em mais três seções, seguidas de uma conclusão. A primeira procura articular a iniciativa de alocação de recursos em saúde representada pelo PAB com os princípios de igualdade e eqüidade subjacentes aos paradigmas de justiça vigentes no debate sobre alocação de recursos públicos em políticas sociais. A segunda apresenta os resultados da análise dos efeitos da introdução do PAB sobre a distribuição de recursos para atenção básica e a prestação de serviços básicos de saúde, no conjunto de municípios de Minas Gerais. A terceira aborda os desafios enfrentados pela esfera estadual e pelos cinco municípios estudados, no processo de implementação do programa de atenção básica.

\section{Igualdade e eqüidade em saúde e a implantação do PAB}

Vem se tornando cada vez mais clara entre estudiosos e formuladores de políticas públicas a importância da análise conceitual das noções de igualdade e eqüidade, como princípios norteadores das decisões sobre alocação de recursos para políticas sociais, particularmente na área da saúde.

De saída, é importante esclarecer que igualdade e eqüidade são, ambos, princípios comprometidos com a idéia de igualdade de direitos entre os indivíduos, que constitui um valor moderno associado ao desenvolvimento do Estado-nação e à expansão contemporânea dos direitos sociais de cidadania. Mas a separação entre os dois princípios se dá na forma como tais direitos são atendidos, o que irá resultar em perspectivas diferentes com relação a regras distributivas (Medeiros, 1999).

No caso particular da saúde, é bem sabido que $o$ acesso a esse bem depende das condições socioeconômicas mais gerais vividas pelos diferentes grupos e setores da sociedade, bem como do acesso a serviços de saúde. Whitehead (1991) aponta o acesso inadequado a serviços como um dos determinantes de desigualdades em saúde. Dos sete determinantes deste tipo de desigualdades especificados pela autora - a) variação natural, biológica; b) comportamento insalubre livremente escolhido; c) vantagens 
transitórias resultantes da adoção gradual de novos recursos de promoção da saúde; d) comportamentos danosos compulsórios; e) exposição a condições deletérias de trabalho e vida; f) acesso inadequado a serviços; g) seleção natural que leve a mobilidade social descendente -, os três primeiros seriam considerados inevitáveis e, conseqüentemente, aceitáveis. Os demais seriam evitáveis e injustos, configurando resultados iníquos em saúde. Assim, para Whitehead (1991), os diferenciais de saúde entre pessoas e/ou grupos seriam iníquos e, portanto, inaceitáveis e injustos quando forem considerados desnecessários e evitáveis.

Numa sociedade como a brasileira, marcada pelas enormes desigualdades sociais, a pobreza massiva gera baixos níveis de saúde e leva à dependência da maioria em relação aos serviços prestados pelo Estado. Neste caso, a alocação de recursos para o atendimento do direito à saúde precisa levar em conta as diferenças que dizem respeito tanto aos níveis de saúde como ao acesso a serviços. Como há grande diferenciação entre as necessidades de saúde de indivíduos e grupos, e entre o seu acesso aos serviços de saúde, igualdade e eqüidade não serão sinônimos. Não se trata, pois, de alocar igual montante de recursos para todos (igualdade), mas de alocar recursos de acordo com diferentes necessidades (eqüidade). E, nas situações em que alguns têm mais necessidades insatisfeitas do que outros, mais clara é a distinção entre os princípios de igualdade e eqüidade.

O critério de igualdade na alocação de recursos implica equivalência, invariabilidade. Já eqüidade remete à idéia de justiça. Igual pode não ser equânime, ou pode ser totalmente equânime. Para que uma situação possa ser considerada eqüitativa é preciso que, simultaneamente, casos relevantemente similares sejam tratados de forma similar (eqüidade horizontal), e casos relevantemente diferentes sejam tratados de maneira diferente (eqüidade vertical).

Numa reflexão sobre a política de atenção básica à saúde em diferentes áreas geográficas de um país tão desigual, o PAB representou iniciativa orientada para a redução da desigualdade, na medida em que rompeu com a lógica dominante de alocação de recursos - pagamento por procedimentos - pelo menos para a atenção básica. Nos termos dessa lógica, os municípios com menor capacidade de oferta de serviços, em geral os mais pobres, recebiam valores muito baixos para a atenção ambulatorial. Este era claramente o caso de Minas Gerais, onde foi constatada uma enorme desigualdade na distribuição de recursos financeiros de origem federal entre municípios, no período anterior ao PAB (Fundação João Pinheiro, 1997).

$\mathrm{Na}$ verdade, a forma como foi concebido o $\mathrm{PAB}$ pode ser analisada como uma iniciativa no sentido de operacionalizar as duas dimensões da eqüidade mencionadas. A suposição de que todas as pessoas precisam igualmente deste tipo de atenção em algum momento da vida, independentemente de onde ou como vivam, justifica a existência de um piso fixo comprometido, portanto, com a eqüidade horizontal. Ao contemplar ainda, por meio de incentivos (PAB variável), estratégias e programas que focalizam grupos ou populações de risco (programas Saúde da Família, Agentes Comunitários de Saúde, Combate a Carências Nutricionais etc.), o PAB estaria respondendo, também, a demandas de eqüidade vertical. Esta segunda concepção teria, relativamente à primeira, maior potencial redistributivo, já que acarretaria alocação de mais recursos para municípios que apresentem maiores necessidades. Aí reside a riqueza da atenção básica e seu potencial transformador sobre todo o sistema público de atenção à saúde.

Adotando o conceito de eqüidade proposto por Whitehead (1991), assim como os critérios formulados por Bambas e Casas (2001) para avaliação da eqüidade/iniqüidade em saúde, considerou-se iníqua a situação prévia à instituição do PAB. Isto porque as diferenças verificadas na distribuição de recursos financeiros não se justificavam, pois: a) não contemplavam preferencialmente os mais necessitados; b) poderiam ser alteradas, dados os recursos técnicos e financeiros disponíveis; c) não refletiam livre escolha da população, notadamente dos setores mais pobres daqueles municípios que contam com menor volume de recursos destinados à saúde, apesar de apresentarem um quadro mais agudo de necessidades; e, d) poderiam ser imputáveis a um determinado agente, no caso, os gestores públicos que, ou não estavam eticamente comprometidos com o princípio de eqüidade, ou ainda não agregavam força política suficiente para inverter prioridades a favor dos portadores de maiores necessidades.

Explicitadas as concepções de igualdade e eqüidade que informam o presente estudo, passa-se à análise dos resultados quantitativos da introdução do PAB. 


\section{Efeitos da introdução do PAB}

Em países caracterizados por extremas desigualdades sociais e econômicas, como o Brasil, ganha relevância o desenvolvimento de processos sistemáticos de monitoramento da distribuição regional de recursos públicos que possam subsidiar políticas de saúde orientadas para a eqüidade.

Vários estudos reconhecem os avanços verificados nas técnicas de mensuração das desigualdades (Dachs, 2001; Kunst e Mackenbach, 1994). No entanto, qualificar essas desigualdades como iniqüidades requer o conhecimento das causas das desigualdades e o julgamento sobre a justeza dessas causas à luz de um conceito de eqüidade previamente explicitado e de sua forma de operacionalização.

Tendo como referência o conceito de eqüidade desenvolvido anteriormente, buscou-se analisar o efeito da introdução do PAB sobre a distribuição dos recursos federais para atenção básica entre municípios de Minas Gerais, utilizando o critério geográfico, pois os dados disponíveis se referem às divisões político-administrativas do Estado. Os valores das transferências federais em 2000 foram confrontados com os recursos federais destinados ao pagamento de procedimentos básicos da atenção ambulatorial em 1997, ano anterior à implantação do PAB. Os dados analisados se referem aos 831 municípios que se encontravam habilitados à gestão plena ao final de 2000 .

Num primeiro momento, foram identificadas as desigualdades na distribuição de recursos federais entre municípios, comparando-se os anos de 1997 e 2000. Para avaliar em que extensão as desigualdades identificadas seriam consideradas iniqüidades, buscou-se num segundo momento correlacionar as desigualdades detectadas com as características dos municípios em termos de sua capacidade de gasto, administrativa e de produção de serviços de saúde. Esse procedimento visava identificar os condicionantes das desigualdades. Em seguida, buscou-se relacionar o gasto com atenção básica às medidas de necessidades propostas neste estudo, o que permitiu um julgamento sobre a orientação das mudanças ocorridas na alocação de recursos após a instituição do PAB.

Visando enfrentar a enorme heterogeneidade entre os municípios de Minas Gerais, buscou-se agrupá-los segundo as dez regiões de planejamento do Estado, o porte populacional, e em grupos homogêneos. Estes foram cons- truídos por intermédio da análise de conglomerados (clusters analysis), a partir de variáveis relacionadas ao desempenho municipal, em termos de capacidade de gasto e administrativa, e ao sistema de serviços de saúde. Dentre os cinco grupos construídos, quatro atendiam ao critério de homogeneidade interna com relação às variáveis utilizadas, ao mesmo tempo que apresentavam diferenças significativas entre si. O quinto agrupamento foi composto por municípios habilitados em gestão plena do sistema de saúde municipal, característica que os diferenciava dos demais quanto ao sistema de saúde.

Comparando o valor total transferido aos municípios mineiros para a atenção básica em 2000 (R \$314,96 milhões) com aquele transferido em 1997 ( $\mathrm{R} \$ 295,67$ em valores constantes de 2000), verifica-se um aumento de 6,5\% para o conjunto dos municípios. A criação do PAB representou um aporte adicional de recursos para 538 municípios (65\% do total) e implicou um aumento do valor per capita médio transferido aos municípios para a atenção básica de $\mathrm{R} \$ 16,02$ em 1997 (a preços de 2000) para $\mathrm{R} \$ 19,28$, em 2000. Ao garantir um mínimo de $\mathrm{R} \$ 10,00$ por habitante/ano, a introdução do PAB representou uma mudança em relação à situação que vigorava em 1997, quando os recursos federais para atenção básica de um quarto dos municípios mineiros foram inferiores a $\mathrm{R} \$ 10,00$, tendo sido registrados valores próximos de zero. O valor do quartil superior também cresceu, passando de $\mathrm{R} \$ 20,09$ para $\mathrm{R} \$ 25,62$. Embora tenha havido uma redução da diferença entre os valores extremos, a variabilidade em torno da média, medida pelo desvio padrão, não se alterou.

Analisando a distribuição dos recursos do PAB segundo as regiões de planejamento do Estado, observou-se que, entre 1997 e 2000, o valor médio dos recursos para atenção básica cresceu em todas as regiões, com destaque para a região mais pobre do Estado, Jequitinhonha/Mucuri, que apresentou uma variação positiva de $57 \%$. Cabe salientar que esta se destacava pelo menor valor médio para o gasto federal ambulatorial em 1997. A elevação dos valores federais médios per capita de todas as regiões ocorreu concomitante à manutenção da variabilidade entre e intra-regiões.

Quando se agruparam os municípios segundo faixas de população, foi observado que a introdução do PAB beneficiou principalmente aqueles de pequeno porte (com até $10 \mathrm{mil} \mathrm{ha-}$ bitantes), que apresentaram maior crescimento 
para o PAB per capita médio (38\%). Como conseqüência, esses municípios aumentaram sua participação entre aqueles com maiores valores per capita para atenção básica. O conjunto de municípios com população superior a 50 mil teve seu valor médio per capita reduzido entre 1997 e 2000 . A variabilidade entre as faixas de população também aumentou, tendo havido discreta redução internamente a elas.

A análise da distribuição dos recursos segundo os cinco grupos homogêneos de municípios indicou que os valores médios para o PAB per capita em 2000 variaram de $\mathrm{R} \$ 18,00$ a $\mathrm{R} \$ 25,80$. Dois grupos apresentaram média acima de 25 reais. No primeiro, composto predominantemente de municípios de porte populacional muito pequeno, de baixo poder econômico, baixa capacidade de gasto e média capacidade administrativa, o valor médio mais elevado refletia a adesão aos programas que integram o PAB variável. No outro grupo, o elevado per capita médio refletia a alta produção de serviços que caracterizava seus municípios, com repercussões sobre os seus valores históricos e, conseqüentemente, sobre o valor médio do PAB fixo de seus municípios que se mostrou superior ao dos demais grupos. Entre 1997 e 2000, houve discreta redução do valor médio do PAB per capita no grupo de municípios em gestão plena do sistema e significativa redução naquele grupo que congregava municípios caracterizados por elevados valores per capita para o atendimento ambulatorial em 1997. Nos demais grupos, houve elevação dos valores médios. Essa constatação evidencia que, em que pese a consideração dos valores históricos da produção ambulatorial na definição do piso de cada município, houve uma redistribuição dos recursos federais para a atenção básica, em detrimento dos municípios com valores relativamente mais elevados.

\section{Influência dos incentivos na composição do valor total do PAB}

A análise dos componentes do $\mathrm{PAB}$ - a parcela fixa e os incentivos da parcela variável - complementou a caracterização das mudanças ocorridas nos valores transferidos para atenção básica aos municípios. Em 2000, a participação da parcela fixa foi, em média, de $65 \%$, tendo variado de um mínimo de $19 \%$ a um máximo de $98 \%$. Ainda que seja significativo o número de municípios que aderiram aos programas propostos pelo Ministério da Saúde, foi bastante variável o efeito dos incentivos na composição do valor total do PAB. As participações do PSF e do PACS variaram de percentuais extremamente baixos (inferiores a $1 \%$ ) a percentuais significativos, como 66\% (PSF) e 32\% (PACS). Destaca-se a importância do PSF, que contribuiu com mais de $32 \%$ do valor do PAB para $50 \%$ dos municípios que aderiram a esse programa.

Analisando a composição do PAB dos municípios agregados segundo faixas de população, grupos homogêneos e regiões de planejamento, observou-se que a participação da parcela fixa foi menor, em termos médios, nos municípios com até 10 mil habitantes, no grupo caracterizado por baixa capacidade fiscal, baixo nível econômico e média produção de serviços, e nas regiões Noroeste e Norte. A parte fixa adquiriu maior importância, em termos médios, na composição do PAB nos municípios de médio porte (entre 50 e 200 mil habitantes), no grupo caracterizado por média capacidade fiscal e de produção de serviços e médio nível econômico, e nas regiões Rio Doce e Sul de Minas.

Considerando apenas a participação dos recursos decorrentes da adesão ao PSF nos valores do PAB, observou-se que ela é maior, em termos médios, no Triângulo, nos municípios de pequeno porte e no grupo caracterizado por baixa capacidade fiscal, baixo nível econômico e média produção de serviços. Nos dois últimos casos, os resultados refletem os mecanismos de financiamento desse programa que contempla, com maiores acréscimos sobre o $\mathrm{PAB}$, os municípios com maior percentual de cobertura da população, favorecendo dessa forma os de pequeno porte.

Comparando os municípios com menores/maiores valores de PAB per capita (1o e 4o quartil), observa-se que a participação da parcela fixa representou, em média, $88 \%$ para aqueles com os menores valores. No caso dos municípios com maiores valores, a participação média da parcela fixa foi bem inferior, evidência de que os valores mais elevados do PAB foram obtidos a partir da adesão aos programas que integram o PAB variável, principalmente ao PSF.

Esses resultados apontam para a relevância de processos de monitoramento que permitam avaliar as conseqüências da introdução de uma política de distribuição igualitária de recursos, com base populacional, associada à promoção 
de incentivos a programas de âmbito nacional. Ao serem implementados de modo não pactuado entre as três esferas, dissociados de instrumentos de coordenação e de informações relativas às necessidades da população, os incentivos federais reforçam uma lógica de atuação autárquica por parte dos municípios, podendo estar sendo interpretados mais como uma forma de captação de recursos do que de redefinição da responsabilidade pela prestação dos serviços básicos de saúde.

\section{Mudanças na prestação de serviços de atenção básica}

No caso de Minas Gerais, procurou-se avaliar até que ponto a mudança no mecanismo de remuneração da atenção básica, com a introdução do PAB, teria alterado a capacidade de produção de serviços dos municípios, reduzindo os diferenciais de cobertura de atenção básica previamente existentes entre municípios e regiões do Estado (Fundação João Pinheiro, 1997).

A partir da seleção de indicadores de oferta (consulta médica básica), de cobertura vacinal por DPT (vacina tríplice contra difteria, coqueluche e tétano) em menores de um ano e de desempenho de programas de atenção básica, elaborados para os anos 1997 e 2000, com base em dados provenientes do Datasus, foram observados os resultados a seguir descritos.

1) Decréscimo na oferta de consultas médicas básicas, entre 1997 e 2000, especialmente em municípios e regiões que apresentavam, desde períodos anteriores, elevadas médias. Esse decréscimo pode estar refletindo as mudanças ocorridas no sistema de registro de informações ambulatoriais, as quais deixaram de ser pré-condição para a remuneração de serviços prestados. Mas também podem ser relacionadas à introdução do $\mathrm{PAB}$, cujo efeito positivo se observou principalmente em pequenos municípios com um certo grau de organização e de autonomia de gestão. Avaliação anterior do programa de atenção básica, realizada para o conjunto do país, apontou também decréscimo no número de consultas médicas básicas e assinalou diferenças nesse comportamento segundo grandes regiões e agrupamentos homogêneos dos municípios brasileiros (Unicamp, 2001). Do mesmo modo, o decréscimo em Minas Gerais apresentou variações segundo os grupos homogêneos de municípios e as regiões geográficas. Em municípios de pequeno porte e de regiões mais pobres, observou-se estabilidade e pequeno incremento do número de consultas. Por sua vez, em conseqüência do decréscimo no número de consultas básicas, houve redução das diferenças internas a agrupamentos de municípios, com contenção de valores extremos e diminuição da variação intragrupo.

2) A cobertura vacinal por DPT segundo regiões, em 1997, encontrava-se relativamente baixa no Norte de Minas (33\%) e no Jequitinhonha/Mucuri (49\%). As demais regiões apresentavam média de cobertura em torno de $70 \%$, alcançando 78\% no Triângulo. Em 2000, aumenta a cobertura em todo o Estado, sendo a mais baixa taxa a do Jequinhonha/Mucuri, de $76,5 \%$. Nesse ano, as médias de todas as regiões ultrapassam $80 \%$, até mesmo no Norte de Minas, que apresentava a pior média em 1997. Duas regiões (Noroeste e Sul de Minas) apresentaram médias acima de $90 \%$, parâmetro estipulado no Pacto da Atenção Básica de 2000. Embora esses resultados possam sugerir que o programa de vacinação de menores de 1 ano encontra-se consolidado na rotina das unidades básicas de saúde, eles também expõem o grau de necessidade ainda a ser suprida nas regiões mais pobres do Estado.

3) Com relação à avaliação do desempenho de programas de atenção ambulatorial básica foi utilizado um indicador relativo a internações hospitalares por condições sensíveis à atenção ambulatorial. Este indicador tem sido utilizado na avaliação de programas de atenção primária, especialmente em estudos que, adotando um enfoque na população, buscam avaliar em que medida programas ou sistemas de atenção primária à saúde, ao atingir suas metas, melhoram a condição de saúde das populações-alvo. Há evidências de que serviços de atenção primária de melhor qualidade estão associados a taxas mais baixas de internações por condições sensíveis à atenção ambulatorial (Starfield, 1998). Supõe-se que disparidades nas internações por certas doenças consideradas de fácil prevenção, ou aquelas que seriam passíveis de diagnóstico e tratamento precoce de modo a evitar a hospitalização, refletem a inadequação da atenção à saúde às necessidades de determinadas comunidades. Em Minas Gerais, observou-se que as regiões mais carentes apresentavam as maiores taxas de internações por essas condições, com conseqüências para seu gasto hospitalar: Jequitinhonha/Mu- 
curi, Noroeste de Minas, Rio Doce e Norte de Minas. A redução observada nas proporções de internações entre 1997 e 2000 foi significativa, porém mantiveram-se as disparidades regionais. A diferença entre a região com os mais elevados índices (Jequitinhonha) e a com menor taxa (Central) passou de 19,08 (1997) a 15,44 (2000). A situação continua desfavorável naquelas regiões que apresentavam as mais elevadas taxas. Isto sugere a persistência de deficiências na atenção básica, assinalando a importância do acompanhamento da evolução desse indicador para a aferição da adequação da atenção à saúde às necessidades de diferentes grupos populacionais. Quando se analisam as internações segundo faixa etária em 2000, os maiores percentuais de internações por condições sensíveis à atenção ambulatorial ocorrem entre crianças ( 1 a 4 anos) e idosos (60 anos e mais). Esse resultado aponta para a possibilidade de redução das disparidades através da melhoria da atenção básica, especialmente orientada para atendimento a esses grupos etários naquelas regiões do Estado. Assim, disparidades nas taxas de internações por condições sensíveis à atenção ambulatorial entre regiões refletem a permanência de inadequado atendimento às necessidades de saúde de diferentes grupos populacionais, constituindo esse indicador importante parâmetro de aferição de iniqüidades em saúde. A atuação voltada para grupos específicos como crianças menores de cinco anos e idosos cria um potencial de redução dessa disparidade.

\section{Associação entre gasto, desempenho municipal e necessidades dos municípios}

Evidenciado o aumento do gasto com atenção básica ocorrido na maioria dos municípios de Minas Gerais, paralelamente à manutenção das desigualdades na distribuição dos recursos, e verificada a importância dos incentivos, principalmente do PSF, buscou-se direcionar a análise para a associação entre gasto, desempenho municipal e necessidades dos municípios.

Num primeiro momento, ajustou-se um modelo de regressão múltipla, em que a variação do gasto com atenção básica entre 1997 e 2000 constituiu a variável dependente. As variáveis explicativas foram desempenho municipal, porte populacional e necessidade em saúde. O desempenho municipal foi medido pelos fatores que sintetizaram a capacidade de gasto, capacidade administrativa instalada e produção de serviços de saúde, determinados para a criação do agrupamento homogêneo de municípios. A medida de necessidade incluída no modelo foi o coeficiente de mortalidade infantil. Os resultados indicaram que apenas os fatores produção de serviços e capacidade de gasto tinham poder de explicar a variação do gasto com atenção básica nos municípios antes e após a instituição do PAB, nos anos estudados. Em decorrência, selecionou-se o modelo de associação entre a variação do gasto e os fatores de produção de serviços de saúde e de capacidade de gasto. Os resultados do modelo selecionado indicaram que a variação do $\mathrm{PAB}$ per capita entre 1997 e 2000 está negativamente associada com a produção de serviços de saúde e com a capacidade de gasto dos municípios em 1997 (Tabela 1). Isto é, a introdução de um piso favoreceu municípios com capacidade de gasto e produção de serviços relativamente menores, apontando para uma tendência de reversão da situação anterior. Esse modelo, entretanto, explica $35 \%$ da variabilidade da variável dependente. Existem, portanto, outros fatores não identificados neste estudo que condicionam as desigualdades nos gastos com atenção básica à saúde.

Num segundo momento, foram associados os valores per capita do PAB 2000 e proxy PAB 1997 com variáveis que expressam necessidades em saúde nos municípios (mortalidade infantil, percentual de domicílios com abastecimento de água, percentual de domicílios servidos de esgoto, proporção de população rural e porte populacional). Essa associação tinha como objetivo verificar se os diferenciais presentes nos valores PAB 2000 per capita refletiam mudanças pró-eqüidade. Entre 1997 e 2000, observou-se uma inversão do sinal do coeficiente de correlação entre os valores de $\mathrm{PAB}$ per capita e as condições sociodemográficas dos municípios ( $\mathrm{Ta}$ bela 2). Por exemplo, o coeficiente de Pearson para a correlação entre as variáveis Proxy PAB 1997 e percentual de domicílios com esgoto é positivo, indicando que maiores gastos se deram em municípios com mais altos percentuais de domicílios com esgoto. Em 2000, o coeficiente é negativo, mostrando que, contrariamente ao que se observou em 1997, os valores per capita do PAB foram maiores em municípios com menores percentuais de domicílios com esgoto, como é desejável em programas orientados para eqüidade. A correlação entre valores $\mathrm{PAB}$ e as outras variáveis - domicílios 
Tabela 1

Estimativa de parâmetros de regressão linear múltipla relacionando a variação do gasto com Atenção Básica entre 1997 e 2000 e variáveis explicativas.

\begin{tabular}{lcccc}
\hline Variável explicativa & \multicolumn{3}{c}{ Coeficientes de regressão para a variável resposta - variação do gasto } \\
PAB entre 1997 e 2000
\end{tabular}

Fonte: Fundação João Pinheiro (FJP). Centro de Estudos Econômicos e Sociais (CEES).

Nota: Sinal convencional utilizado: (..) não se aplica dado numérico.

Tabela 2

Coeficientes de correlação entre proxy PAB 1997, PAB 2000

e variáveis sociodemográficas municipais - Minas Gerais - 1997/2000.

\begin{tabular}{lcccc}
\hline Variável & \multicolumn{2}{c}{ PROXY PAB 1997 } & PAB 2000 \\
\hline & $\begin{array}{c}\text { Coeficiente } \\
\text { de Pearson }\end{array}$ & $\begin{array}{c}\text { Nível de } \\
\text { significância }\end{array}$ & $\begin{array}{c}\text { Coeficiente } \\
\text { de Pearson }\end{array}$ & $\begin{array}{c}\text { Nível de } \\
\text { significância }\end{array}$ \\
\hline $\begin{array}{l}\text { Domicílios com abastecimento } \\
\text { de água (\%) }\end{array}$ & 0,205 & 0,000 & $-0,024$ & 0,493 \\
$\begin{array}{l}\text { Domicílios com esgoto (\%) } \\
\text { Faixas de população }\end{array}$ & 0,096 & 0,005 & $-0,153$ & 0,000 \\
População rural (\%) & 0,104 & 0,002 & $-0,191$ & 0,000 \\
Coeficiente de mortalidade & $-0,179$ & 0,000 & 0,100 & 0,004 \\
infantil & $-0,107$ & 0,002 & $-0,039$ & 0,262 \\
\hline
\end{tabular}

Fonte: Fundação João Pinheiro (FJP). Centro de Estudos Econômicos e Sociais (CEES).

com abastecimento de água, proporção de população rural e porte populacional - apresentou comportamento similar, embora nem todas tenham alcançado significância estatística. $\mathrm{O}$ resultado para a correlação entre os valores per capita do gasto PAB e o coeficiente de mortalidade infantil não apresentou o mesmo comportamento. Embora não tenha havido uma inversão de sinal, a mudança verificada foi no sentido de uma menor relação inversa em 2000, tendo o coeficiente de Pearson se aproximado de zero e, portanto, tendido para uma relação positiva, como seria desejável.

Os resultados da análise da implementação do PAB em Minas Gerais, que se deu em condições de extrema desigualdade na distribuição dos recursos federais para a atenção básica, apontam uma tendência de reorientação na alocação de recursos no sentido de uma distribuição menos injusta. A continuidade do processo, no entanto, requer a adoção de critérios que, explicitamente, levem em consideração as necessidades diferenciadas de saúde.

\section{Desafios enfrentados pelo Programa de Atenção Básica em Minas Gerais}

A presente seção trata dos aspectos institucionais e organizacionais da atenção à saúde presentes na esfera administrativa estadual e em cinco municípios selecionados e de suas influências na implementação do programa de atenção básica. 


\section{A política de atenção básica da esfera estadual}

Entre 1991 e 1994, a SES/MG desencadeou um programa de municipalização do atendimento que consistiu na transferência progressiva da prestação de serviços para a esfera municipal, especialmente a rede básica de serviços. Como resultado desse esforço, em meados de 1995, 92\% dos 756 municípios mineiros então existentes encontravam-se habilitados em alguma forma de gestão.

Entretanto, o avanço do processo de municipalização dos serviços não teve correspondência na reorganização da SES. Esta se manteve, com poucas alterações, uma organização com estrutura e métodos concebidos para operar serviços de atenção primária seletiva para segmentos sociais de baixo poder econômico e político, inadequada para o desempenho de atribuições como a regulação geral do sistema estadual, incluindo a macroalocação de recursos; apoio e assessoria técnica aos municípios; promoção da integração entre os gestores municipais, com vistas à organização de sistemas funcionais e resolutivos em cada região. Tampouco as Diretorias Regionais de Saúde (DRS), anteriormente concebidas para a supervisão da operação direta de serviços, lograram reciclarse para atender às exigências de novos papéis institucionais.

O esvaziamento da esfera estadual acabou por fragilizar a SES, retirando-lhe instrumentos materiais de expressão de poder político (notadamente recursos financeiros) e diminuindo sua legitimidade perante os vários atores com interesses diversificados e conflitantes - prestadores privados, governos municipais, corporações profissionais, organizações nãogovernamentais - que passam a atuar na arena político-institucional detendo poderosos instrumentos de pressão.

O governo do período 1995-1998 não atuou no sentido de modificar o quadro descrito. $\mathrm{Na}$ área da atenção básica, foi assumida como prioridade a ampliação do Programa Saúde da Família (PSF) e do Programa de Agentes Comunitários de Saúde (PACS).

Para enfrentar a questão da oferta de atenção secundária e terciária, os gestores estaduais priorizaram a expansão para todo o estado do consórcio de municípios. Mas, mesmo havendo sido assumidos como política de governo no período, os consórcios intermunicipais de saúde constituíram, como o próprio nome in- dica, uma organização exclusiva de municípios. Além da fragilidade da esfera estadual no financiamento dos consórcios, chama a atenção o fato de que quase não houve articulação de suas atividades pelas instâncias regionais do setor, as DRS (Fundação João Pinheiro, 1996).

O período 1999-2002 é marcado pela preocupação dos gestores estaduais em revitalizar os instrumentos de planejamento para a retomada dos princípios de regionalização e hierarquização dos serviços de saúde, em consonância com a Norma Operacional de Assistência à Saúde NOAS 01/2001. Esta, como é sabido, além de prever a ampliação das responsabilidades dos municípios com relação à atenção básica, busca corrigir distorções da chamada municipalização autárquica, criando mecanismos de fortalecimento da gestão estadual e atribuindo às secretarias estaduais a responsabilidade pela coordenação e pactuação, com vistas à organização de redes de assistência resolutivas.

Em 2001, a partir de resolução da CIB-MG, é elaborado pela SES, com a participação do Conselho de Secretários Municipais de Saúde (COSEMS) e do Conselho Estadual de Saúde (CES), o Plano Diretor de Regionalização (PDR). Além das instâncias previstas na NOAS - município em gestão plena da atenção básica ampliada, módulos, e microrregiões de saúde -, o modelo de regionalização da SES discrimina região assistencial, macrorregião e polarização estadual, como níveis complementares de agregação tecnológica.

No nível central, foi instituída informalmente a Diretoria de Atenção Básica, no âmbito da Superintendência Operacional de Saúde, o que revela preocupação com o desenvolvimento da atenção básica, que teria sido mais enfatizada após a implantação do PAB, e do Pacto de Atenção Básica como condição necessária para habilitação nos termos da NOAS.

O reordenamento do modelo de organização - municipalização inserida no processo de regionalização/hierarquização, buscando racionalizar o uso de recursos - constitui, também, diretriz do Plano Estadual de Saúde da SES para 2001, que propõe mudanças do modelo assistencial a favor da atenção básica. Da mesma forma, ocorreu no período grande expansão do PSF/PACS no Estado: em mais de 300 municípios mineiros o PSF atingiu cobertura superior a 70\%; em outros tantos está presente, embora com índices menores de cobertura, e enfrentando dificuldades para contratação de pessoal treinado. 
A elaboração do PDR constituiu um processo marcado por grandes conflitos entre os representantes do nível central, particularmente da Diretoria de Planejamento, e dos demais níveis do SUS estadual. A mesma situação de conflito marcou a atuação da esfera estadual em seu esforço de desenvolver, junto com as DRS e os municípios, e seus organismos colegiados - CIB e COSEMS regionais e estaduais -, a Programação Pactuada Integrada (PPI) e o Plano Diretor de Investimentos (PDI). Em tais disputas, ficou clara a baixa legitimidade da SES para negociar a elaboração e implementação dos instrumentos de gestão compartilhada (PDR, PDI, PPI). Além do não-cumprimento pela SES de seu papel de co-financiador do SUS, seu esvaziamento técnico, ocorrido com a municipalização, e sua carência de equipamentos necessários à produção e organização de informações teriam comprometido o desempenho do nível estadual na alocação de recursos fixação de tetos municipais, no âmbito da PPI, mediando disputas entre municípios, com vistas a uma distribuição mais racional e eqüitativa de recursos.

Busca-se, a seguir, examinar mais concretamente os efeitos da instituição do PAB para a esfera municipal no contexto de esvaziamento técnico, político e financeiro das instâncias estadual e regionais de saúde, brevemente descrito.

\section{A experiência de organização da atenção básica pela esfera municipal}

Serão aqui considerados alguns aspectos do programa de atenção básica, a partir da experiência de cinco municípios cuja escolha foi intencional, não tendo pretensão de representatividade estatística.

São os seguintes os municípios incluídos no estudo: Aricanduva, Caeté, Francisco Badaró, Itambacuri e Peçanha, cada um pertencente a um dos cinco agrupamentos criados para a análise quantitativa. Com exceção de Caeté, localizado na Região Metropolitana de Belo Horizonte, todos os demais se situam no Nordeste do Estado, em microrregiões com alta necessidade em saúde (Vales do Jequitinhonha, $\mathrm{Mu}$ curi e Rio Doce).

Aricanduva e Francisco Badaró são municípios de pequeno porte (até 10 mil habitantes), predominantemente rurais. Peçanha tem população inferior a 20 mil habitantes, pouco mais da metade na área rural. Os outros, com população entre 20 e 40 mil, possuem elevado percentual de população urbana. Todos apre- sentavam valores do PIB per capita inferiores à média do Estado em 1998.

As condições de saneamento nos municípios estudados são similares: melhores serviços na sede que nos distritos, e população rural pouco atendida. Correlatamente, doenças infecciosas e parasitárias, entre elas a esquistossomose, ainda são endêmicas nos municípios de Aricanduva, Itambacuri e Peçanha. E o coeficiente de mortalidade infantil estimado para 1998 variava de 49 (Itambacuri), 41 (Peçanha), 37 (Aricanduva e Francisco Badaró) a 29 (Caeté) mortes de menores de 1 ano por mil nascidos vivos.

Em todos, são altas as taxas de internações hospitalares por condições sensíveis a atenção ambulatorial (acima de 30\%). Apenas Caeté realizou mais de 1,5 consulta médica básica por habitante/ano em 2000, assim como recebeu valores do PAB per capita acima da média do Estado. E somente Itambacuri encontrava-se em gestão plena do sistema de saúde, os demais enquadrando-se na gestão plena da atenção básica.

Verificou-se, nos municípios selecionados, aumento da rede após a municipalização. Em Caeté, a expansão se deu paralelamente à reorientação do modelo de atenção básica através do PSF, iniciado em 1994. Em Aricanduva e Itambacuri, a adesão ao PSF é mais recente, claramente motivada pela instituição de incentivos federais. Já Francisco Badaró e Peçanha desenvolveram a estratégia do PACS, voltada para o atendimento básico a populações dispersas nas áreas rurais. Em Aricanduva, o sistema de atenção à saúde se restringe a unidades básicas.

Num primeiro momento, os cinco municípios se beneficiaram com a implantação do PAB. O ganho foi mais significativo para Aricanduva, que passa de $\mathrm{R} \$ 5,71$ per capita, em 1997, ao PAB de R $\$ 16,16$, em 2000. Posteriormente, o ganho inicial de recursos irá conduzir à necessidade de ampliação do gasto com recursos próprios, como será visto adiante.

Os incentivos federais para o desenvolvimento dos programas de atenção básica que compõem a parte variável do PAB passaram a ser buscados pelos municípios estudados numa tentativa de consolidação da atenção básica: a parcela variável significava, já em 2000, acréscimo de $\mathrm{R} \$ 4,80$ (Aricanduva), de $\mathrm{R} \$ 6,10$ (Francisco Badaró), de R $\$ 6,84$ (Itambacuri) e de $\mathrm{R} \$ 3,30$ per capita (Peçanha). Caeté se destacava pela maior parcela variável, que correspondia a $\mathrm{R} \$ 18,20$ per capita. 
Para os municípios, a implantação desses programas muitas vezes correspondeu à incorporação de um modelo de intervenção sem flexibilidade para adaptação às realidades locais. A falta de autonomia para programar e avaliar as ações desenvolvidas no âmbito dos programas, assim como a fragmentação do financiamento imporia "amarras" à utilização dos recursos. E, mesmo em municípios localizados próximo à capital do Estado, como é o caso de Caeté, os gestores municipais encontram dificuldades para a manutenção de médicos e enfermeiros integrados ao PSF.

$\mathrm{O}$ aumento da demanda gerado pela reorganização da atenção básica criou pressão sobre o sistema de referências para a atenção secundária e terciária. O atual sistema de cotas de atendimento atribuídas aos municípios por um mecanismo de pactuação entre gestores, mediada pelas instâncias regional e estadual, temse mostrado insatisfatório e os gestores municipais - pressionados pela demanda local - acabam aplicando recursos próprios, comprometendo a receita disponível do município. Quatro dos municípios visitados buscam ampliar sua capacidade de oferta de assistência especializada participando em Consórcios Intermunicipais de Saúde. Essa participação representa, no entanto, grande ônus para os municípios, que dispõem para os consórcios de um percentual, entre 1 a 1,5\%, do Fundo de Participação dos Municípios (FPM). De fato, todos os municípios estudados aplicavam em saúde mais de $12 \%$ da receita própria. Francisco Badaró e Itambacuri gastaram, respectivamente, 22\% (2002) e 31\% (2001).

Mas a despeito das dificuldades apontadas, já é possível identificar avanços em ações de saúde relacionados ao PAB. Destaca-se a ampliação significativa da cobertura por vacinas tríplice e BCG nos quatro municípios da região Nordeste do Estado, onde era muito baixa em 1997. Nos termos do Pacto de Indicadores da Atenção Básica, os cinco municípios estudados se comprometeram com a redução do número de óbitos de menores de um ano; aumento da cobertura vacinal; melhoria do atendimento pré-natal; controle de casos de hipertensão e diabetes e prevenção dos agravos decorrentes, reduzindo taxas de internação; controle da tuberculose e hanseníase, incluindo busca ativa de casos novos; realização de procedimentos odontológicos coletivos na faixa etária de 0 a 14 anos. Parece, pois, inegável a inclusão das ações de atenção básica na agenda política dos gestores municipais.
A breve análise da política de atenção básica do governo estadual, bem como das atividades desenvolvidas pelos municípios após a instituição do PAB, propiciou o reconhecimento das seguintes questões que ainda desafiam os gestores de saúde.

A primeira se refere ao financiamento do sistema. Insuficiência quantitativa de recursos, baixo nível de participação da esfera estadual e distribuição desigual de recursos entre regiões e municípios têm afetado sobremaneira a organização do sistema de saúde, especialmente do atendimento básico.

Ainda como desafio coloca-se o fortalecimento da capacidade de coordenação da esfera estadual de governo. A ação municipal se encontra limitada pela insatisfatória articulação dos níveis assistenciais e pelo ainda incipiente processo de regionalização da assistência à saúde. Sem a efetiva participação das instâncias regionais e estadual, difícil será promover o compartilhamento de serviços especializados entre municípios e a organização de um sistema de referência e contra-referência adequado às necessidades locais.

\section{Conclusão}

O estudo permitiu avaliar as mudanças na distribuição de recursos financeiros entre municípios de Minas Gerais e na prestação de serviços básicos de saúde, após a instituição do PAB.

Em princípio, o estabelecimento do $\mathrm{PAB}$ garantiria igualdade de recursos para atenção básica por meio da alocação com base no critério populacional, promovendo assim eqüidade horizontal. Ao contemplar, mediante incentivos, estratégias e programas focalizados em populações de risco, o PAB buscaria responder a demandas de eqüidade vertical. No entanto, a forma como foram implementados os incentivos, de modo não pactuado entre as três esferas de governo e dissociados de instrumentos de coordenação, favoreceu a permanência da lógica de atuação autárquica. Ao agir de modo isolado, os municípios tendem a aderir aos programas oficiais movidos, principalmente, pelo interesse de obter mais recursos para a atenção básica. No entanto, os resultados alcançados pelos cinco municípios estudados, tanto em termos de impacto na prestação de serviços quanto da adesão a metas definidas na PPI, são indicadores de maior compromisso dos gestores municipais com a atenção básica. 
O trabalho permitiu constatar que a introdução do PAB foi acompanhada de elevação dos recursos federais transferidos para atenção básica para $65 \%$ dos municípios do Estado. Observou-se que a distribuição dos recursos federais entre municípios se manteve desigual, mas que as mudanças favoreceram os de pequeno porte populacional e o Vale do Jequitinhonha, a região mais pobre do Estado. As diferenças entre os valores per capita recebidos pelos municípios expressam a adesão diferenciada aos programas propostos pelo governo federal e também o fato de terem sido considerados os valores históricos da produção ambulatorial na definição do piso de cada município.

A variação dos valores per capita das transferências federais para atenção básica entre 1997 e 2000 mostrou-se negativamente associada com a produção de serviços de saúde e com a capacidade de gasto dos municípios em 1997. Ou seja, a introdução de um piso favoreceu a reversão de uma situação que privilegiava municípios com maior capacidade instalada e de gasto. A correlação entre os valores municipais do PAB per capita de 1997 e 2000 e as variáveis que refletem necessidades em saúde mostrou, por sua vez, que as mudanças introduzidas tenderam a beneficiar os municípios com maior necessidade em saúde, podendo ser consideradas pró-eqüidade.

Do ponto de vista das mudanças nos serviços, apesar do caráter preliminar da análise, que se refere ao primeiro ano da efetiva implantação do $\mathrm{PAB}$, constatou-se que houve pequeno incremento do número de consultas médicas básicas em municípios de pequeno porte e de regiões mais pobres, especialmente os que atingiram certo grau de organização e autonomia de gestão. Observou-se, ainda, diminuição da variação do número de consultas por habitante no interior dos agrupamentos estudados (grupos homogêneos de municípios, regiões de planejamento e faixas populacio- nais). A partir da avaliação da cobertura vacinal em menores de 1 ano, observou-se que as melhorias alcançadas entre 1997 e 2000 foram significativas, porém revelaram necessidades a serem ainda atendidas nas regiões mais pobres do Estado. Disparidades nas taxas de internações por condições sensíveis à atenção ambulatorial entre regiões refletiram, por sua vez, a permanência de inadequado atendimento às necessidades de saúde de diferentes grupos populacionais. Uma orientação voltada prioritariamente para grupos específicos - como crianças menores de cinco anos e idosos -, na linha dos programas especiais de atenção básica, acarretaria redução da disparidade a favor de portadores de maior necessidade em saúde.

A insuficiência quantitativa de recursos, o baixo nível de participação da esfera estadual diante da municipal no financiamento da saúde, e a distribuição ainda desigual de recursos federais entre regiões e municípios de Minas Gerais têm afetado a organização do sistema de saúde, especialmente do atendimento básico à saúde. Nesse contexto, os maiores desafios enfrentados pelos gestores públicos são o fortalecimento da capacidade de coordenação entre esferas de governo e a recuperação da capacidade de investimento, de modo a reduzir as desigualdades no acesso aos serviços de saúde que são desfavoráveis a regiões, municípios e grupos sociais portadores de maiores necessidades.

A adoção de um modelo de alocação eqüitativa de recursos, que contemple de forma sistemática a discriminação positiva dos municípios com maiores necessidades em saúde, representaria um avanço relativamente à redistribuição de recursos promovida pelo PAB. Daí a importância de se promover, nos níveis federal e estadual de governo, uma reflexão sobre a necessidade de revisão da fórmula $\mathrm{PAB}$, de modo a incorporar os diferenciais relativos de necessidades de saúde dos municípios, concomitante a uma maior presença da esfera estadual. 


\section{Referências bibliográficas}

Bambas A \& Casas JA 2001. Assessing equity in health: conceptual criteria, pp. 12-21. In Pan American Health Organization. Equity and health: views from Pan American Sanitary Bureau. Washington (DC). (Occasional Publications, n. 8).

Bodstein R 2002. Atenção básica na agenda da saúde. Ciência \& Saúde Coletiva 7(3):401-412.

Dachs N 2001. Inequidades en salud: como estudiarlas, pp. 84-104. In H Restrepo \& H Málaga (eds.). Promoción de la salud: cómo construir vida saludable. Editorial Médica Panamericana, Bogotá.

Datasus 2002. Informações de saúde. Disponível em $<$ www.datasus.gov.br >. Acesso em março.

Fundação João Pinheiro 1996. Centro de Estudos de Políticas Sociais. Avaliação dos Consórcios Intermunicipais de Saúde das Microrregiões do Alto do Rio Grande e do Alto Rio São Francisco. Belo Horizonte.

Fundação João Pinheiro 1997. Centro de Estudos Econômicos e Sociais. Gasto federal com assistência à saúde em Minas Gerais: um estudo sobre a desigualdade na distribuição dos recursos financeiros. Belo Horizonte.
Fundação João Pinheiro 2003. Centro de Estudos de Políticas Sociais. Atenção Básica à Saúde em Minas Gerais: desigualdades na distribuição de recursos financeiros e na prestação de serviços básicos após a introdução do Piso de Atenção Básica (PAB). Belo Horizonte.

Kunst AE \& Mackenbach JP 1994. Measuring socioecomic inequalities. Copenhagen: World Health Organization, Regional Office for Europe, Copenhague.

Medeiros M 1999. Princípios de justiça na alocação de recursos em saúde. IPEA, Rio de Janeiro. (Texto para Discussão, n. 687).

Starfield B 1998. Avaliação de programas e sistemas de atenção primária: um enfoque da população. Fundação Ezequiel Dias, Escola de Saúde de Minas Gerais, Belo Horizonte.

Unicamp 2001. Núcleo de Estudos de Políticas Públicas. Avaliação da descentralização dos recursos do Ministério da Saúde para a Atenção Básica: PAB, programas e incentivos. Campinas.

Whitehead M 1991. Los conceptos y principios de la eqüidad en la salud. Organización Panamericana de la Salud, Washington (DC).

Artigo apresentado em 15/9/2003

Aprovado em 30/9/2003

Versão final apresentada em 19/11/2003 